\title{
Implementing Remote Audio as a Diagnostics Tool for Maritime Autonomous Surface Ships
}

\author{
Tychonas Michailidis \\ Birmingham City University \\ Birmingham, United kingdom \\ tychonas.michailidis@bcu.ac.uk \\ Christopher Barlow \\ Solent University \\ Southampton, United kingdom \\ christopher.barlow@solent.ac.uk
}

\author{
Gordon Meadow \\ Seabot XR Ltd \\ Wickham, United kingdom \\ gordon.meadow@seabotxr.com \\ Eshan Rajabally \\ Rolls Royce Ltd \\ Derby, United kingdom \\ eshan.rajabally@rolls-royce.com
}

\begin{abstract}
The role of marine engineers is to maintain the operational state of all systems in the engine room such as to diagnose and rectify problems arising, and to understand what maintenance will be required to keep the vessel appropriately operational and safe. Through training and experience, the engineering crew can read and interpret engine room instrumentation, and employ their intuitive feel for normal operation in-situ. In order to examine the possibilities of future remote and autonomous uncrewed vessels, we developed a virtual reality simulated engine room based on a real vessel. Even though the end product is effectively a virtual simulation, the original audio from the engine room was recorded and used, thus providing a more accurate and immersive experience to the users. This paper examines the use and application of a remote server to feed audio and other data in the simulated virtual engine and to create hypothetical failures scenarios for testing and training. Experience engineers carried out different failure scenarios as they usually do onboard and provided valuable feedback. User testing suggests that upcoming paradigms of the Internet of Audio Things can become a vital element in future operations of Maritime Autonomous Surface Ships.
\end{abstract}

\section{INTRODUCTION}

In the past few years, the possibility of remote and autonomous ships push forward a wave of research concerning the practicality and application. Maritime Autonomous Surface Ships, also known as (MASS), opens new opportunities for the maritime industry, including the roles of shipping companies, shipbuilders and technology systems providers. Many systems onboard are to facilitate and serve the crew (for example the maintaining of fresh drinking water), and with their potential removal, we can simplify the entire ship and improve reliability and productivity while reducing build and operating costs. The leading causes in maritime accidents are because of human intervention; thus autonomous or remotely operated vessels have the potential to reduce accidents and improve safety [2]. Towards the realisation of MASS and other remote and autonomous solutions, there are several practical and technical in nature questions as well as questions relating to operations, legal, safety and the maintenance of mechanical systems on an uncrewed vessel [7]. For instance, the propulsion system is considered critical, and if a failure occurs could endanger the vessel [1]. How can we make a system reliable that such a failure never happens?

Engine rooms are complex systems whereby most of the machinery are linked, co-depended and affecting each other. A fault within the engine room will most likely cause a chain reaction of other possible alarms and failures that engineers can trace back and address the issue. Alarms in the engine room identify non-normal conditions and operations, based on a given threshold. An alarm could lead to potential failures but is not adequate to provide a complete picture or pinpoint what might have caused a failure (see Fig. 1.) There is an urgent need to provide a standardisation of new technologies, and in particular with the current advancements around the Internet of Sounds, that would enable better integration and to provide high standards of user experience and safety [12], [3].

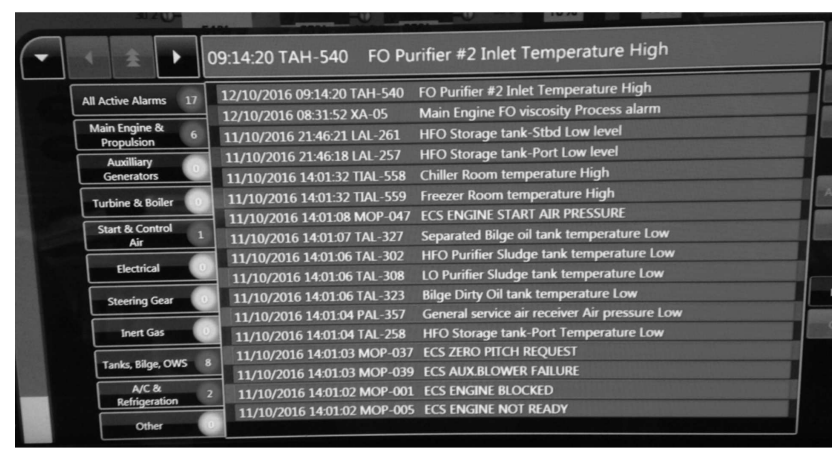

Fig. 1. Alarm display in the engine room providing timestamp, system ID and description of the alarm. The alarms are not prioritised in terms of critical or importance but simply grouped according to subsystems they function.

\section{A. Engineer-centred Approach}

The amount of engineers required onboard depends mainly on the size of the vessel, registered flag, and where and how it operates. Usually, there is a Chief Engineer accompanied by a team. Their task is to maintain the vessel's systems in an operational state, to diagnose and rectify problems arising, and to understand what maintenance will be required to keep the vessel appropriately operational and safe. Marine 
engineers intuitively develop the skill to employ feedback from their senses, and system's data to read and understand how machinery and the whole engine room behaves and responds. Sound and vibrotactile information is an invaluable tool for initial detection of faults by engineers not only in the maritime industry but in other engineering professions [13][15][16]. All machinery in the engine room with moving parts such as pumps, filters, generators, and main engine have a unique audio signature which changes depending on the load, the weather and when potential failure is imminent. Increased friction, insufficient lubrication can result in changes of frequency, knocking sounds, whining, squeaking or arrhythmic sounds, all of which have a distinctive sound quality [4]. Through experience and constant exposure, an engineer learns to decode this rich source of audio-vibration information intuitively, and it subconsciously becomes part of their diagnostics ritual and inspection. While it may not always allow an engineer to diagnose the cause of failure only by listening, it is particularly useful in identifying and narrowing down the source of a problem as well as confirming the validity of known data. Engineers commonly report not fully trusting the control data due to regular false alarms from systems or wrong readings from the sensors [5].

Despite advances in monitoring and diagnostics technology, onboard engineers still rely on their intuitive feel and senses to assess system health or confirm control data before system failure is imminent. The use of audio as a diagnostic tool enables engineers to identify the state of machinery, movement of liquid within the pipes and to access the condition of a component through variations in sound level and pitch. However, due to excessive loudness within the engine room, often above $100 \mathrm{db}$, engineers are required to wear ear defenders. As a result, potential details of audio features get lost. The ability to listen to the sound of the engine room from a remote location and without reduced level can play a significant role as a diagnostics tool in the potential crewless vessel scenario. Internet of Audio Things paradigm proposes a novel approach where audio features from every device in the engine room can be potentially recorded, analysed and transmitted to a control room [3]. Engineers can enhance audio information by isolating the audio of selected machinery from the extensive background noise and listen to specific parts or sections of a large component (e.g. main engine). Remote operations could benefit from examining audio features, gain a broader understanding to support their diagnostics and required actions. For example, a sonification-based system identifies acoustic abnormalities in the manufacturing process [14]. Machine learning algorithms are running on remote cloud to alert operators in real-time about imminent failures and issues. While this research benefits from the ability to process and analyse many audio sources at the same time, in a MASS, the size and speed of transmitted data should be under consideration.

\section{B. Real-Word Data}

The primary challenge of Project IMAGINE (Innovation in MArine enGINEering diagnostics and feedback) was to understand and measure how the engineering crew uses sensory feedback to characterise the state of the vessel while onboard, for both normal and abnormal conditions, and to assess the mechanisms by which this tacit information may be communicated and interpreted effectively to a remote location through Virtual Reality (VR) and other immersive tools [10].

Computer simulations have the potential to immerse remote operators concerning the system in question. Maritime, military and aerospace industries have been using computersimulated systems for operator training, including navigation and bridge systems [6]. Current VR systems have now become readily available to implement them in a wide range of industries apart from gaming, offering a low-cost, large-scale simulation. Their potential for the presentation of real-time data is already being explored [8]. However, sound, vibration, heat and smell, these are not commonly replicated effectively in simulations to enable the Sense of Self-Location [9].

The use of simulated data, including a non-realistic visual representation of engine room, data such as temperature and pressure and audio, would potentially have resulted in a gamelike scenario with unrealistic experience and results. The ferry company Red Funnel granted access to the team to collect data from the engine room of a Raptor Class ro-pax ferry that operates daily services between Southampton and Isle of Wight, in the United Kingdom, with a round-trip duration around two hours. We were able to get the schematics of machinery layout, photographs, audio recordings as well as real data values from pressure and temperature of relevant components in the engine room for the development of the prototype. The simulated VR engine room is $1: 1$ ratio of the real engine room.

In this paper, we present the project and the prototype VR system. Real audio and data are feed in the simulated engine room remotely through a server. A usability test is carried out by experienced marine engineers examines through different scenarios how they can identify the potential cause of failure remotely. The remainder of this paper is organised as follows. Section two presents the methodology; section three examine the server and audio implementation; section four and five goes through the user testing and results, and section six the conclusion.

\section{Methodology}

Audio from machinery within the engine room can enable engineers to diagnose current and potential issues. However, high sound pressure makes it hard to distinguish and focus on details within the engine room. Sound pressure levels measured value of $106 \mathrm{dBL} L_{\text {Aeq, } 15}$ using the NTi XL2 Acoustic Analyzer. With the ability to collect real audio data from all machinery in the engine room, we were able to have a realistic representation of the audio experience.

The general background sound of the engine room was captured with a Soundfield SPS200 placed in key positions. To capture airborne sound with the maximum rejection of spill from other components, we used the Rфde NTG2 shotgun microphone placed around $20 \mathrm{~cm}$ from the source. We also use the Cold Gold directly from different locations of each machinery (Fig.2). These microphones are electronic stethoscopes in which the electret transducer is embedded in a silicone cup, enabling direct connection to machinery while ensuring very high levels of rejection of spill. Onboard audio was recorded using a A Zoom F8 field recorder at 24-bit, $96 \mathrm{kHz}$ resolution. 

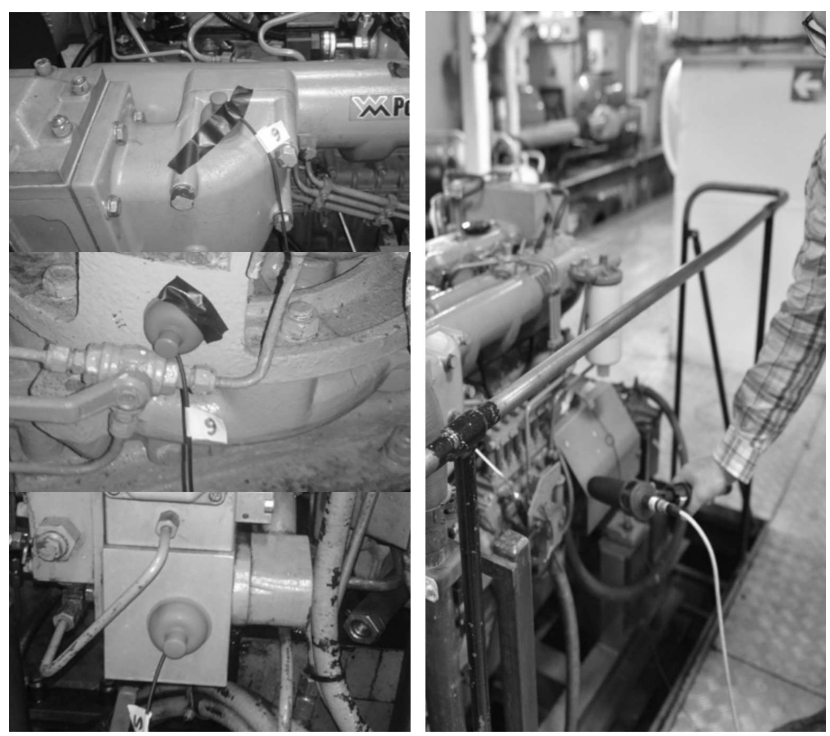

Fig. 2. Left-hand side set of pictures show different machinery recorder with a specialist contact microphone. Right pictures, the audio recording of a generator with a high directional microphone.

The project was developed on the Unreal Engine 4 running on a PC using HTC VIVE Pro HMD system. All audio recordings (contact mics, shotgun and general background) are appropriately positioned in the simulation and rendered binaurally using the HRTF plugin in UE4. A qualitative methodology was applied to gain a broader picture of the user experience and approach when identifying faults. Users complete background demographics and consent form before the test. After going through the usability test, participants took part in a semi-structured interview and asked to complete a post-test User Experience Questioner (UEQ) [17]. The duration of each test was, on average, 75 minutes.

\section{A. Simulating Sound-Vibration Failures}

It was not feasible to record any failure of machinery as the vessel was operating, and we would need to wait for a piece of machinery to fail to capture the audio. Audio of similar system failures on isolated components were sourced through recordings from the engineering workshop at Warsash Maritime Academy and audio from sound libraries where the recording was not possible. Examples include cavitation for each pump, misfiring of the main engine and the generator, single phasing of the compressor, air bleeds from the compressor, and damaged bearings due insufficient lubrication.

Sound files were then convolved with a filter based on the inverse of the frequency response of the contact microphone, thus having a similar frequency response from both signals. The approach ensures that the simulated failure characteristics blended effectively with the original audio and maintaining their unique audio signature. The failure sound and the clean audio were put together to achieve a sound which was recognisably the same machinery, but with a characteristic of the sound associated with a particular mechanical failure. Fig. 3 shows the average spectrum for a clean (healthy) pump sound, and the simulated of a cavitating pump.

In order to make sure that the simulated audio failures of machinery maintain an accurate representation of the actual failure, we present the sounds to five experienced Chief Engineers from the Warsash Maritime Academy. The selected sound failures include a combination of recorded and non recorded audio failures. For this informal evaluation, sounds where presented to the engineers through the early development of the VR system. The engineers listen to the sounds through the build-in headphones of the HTC VIVE Pro. Engineers were asked to rank four different sound versions of each proposed failure and provide an explanation on how they came to that conclusion. This includes audio failure types cavitation, bearing, rotor, jamming, misfire and knocking. The sound files which were considered by the engineers to be the most accurate representations of particular mechanical failures were put forward for the final simulator.

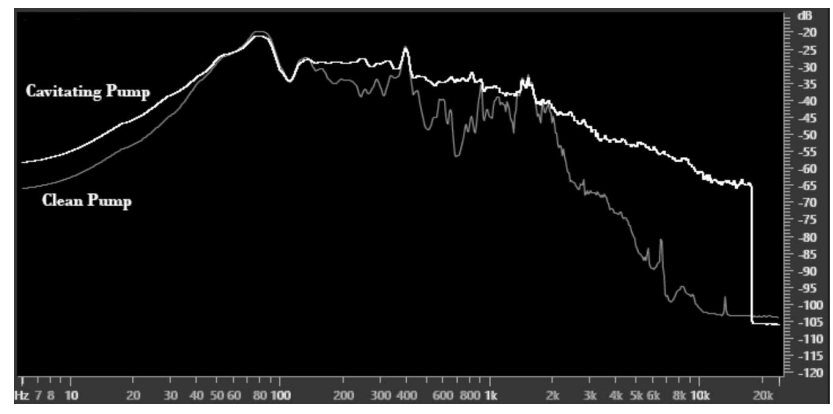

Fig. 3. Average frequency spectrum for the clean and cavitating pump sound

Due to the wide range and size of machinery with moving components, vibrations is an interconnected feature of any engine room. The vibration is re-radiated across the engine room floor and the whole vessel. As a result the way a machinery is vibrating, reflects to its performance. All engineers, during the preliminary interviews, reference how vibrations from the overall engine room and individual machinery plays a significant role in the process of identifying the cause of failure. When a piece of machinery fails, or failure is imminent, the vibrations might have stopped entirely, or there might be a significant change in the intensity and frequency. During the initial review of the prototype with the engineers, they point out the missing vibrotactile feedback experience that is present in the engine room. It was necessary to introduce a vibrating feel into the simulation to make the experience more realistic and increase the level of immersion. Several different options were considered; however, the majority of vibrationbased systems require the user to be seated. A body-worn bass transducer, Subpak M2, was used to apply the vibrations. The user wears the backpack that uses lower frequencies of the audio signal to drive the vibrations. Even though the vibrotactile feedback is not an exact representation of the real vibrating experience, it was vital to provide this mode of feedback during the testing.

\section{SERVER AND REMOTE AUDIO IMPLEMENTATION}

A website interface hosted on a remote server provides the audio and data for the simulation. The server enables the use of real data and audio as if they are streamed from a real uncrewed vessel into a control centre where an engineer will experience remotely. The server feeds data and audio to the simulation in real-time. Graphics elements run locally on the 
PC and thus reducing the processing power required. Data, including audio, changed according to the failure scenarios presented for the user testing while the user is in the simulation minimising any distraction. The interface provides a list of all available machinery and associated components of the engine room that the administrator can control.

Due to the variety of interconnected and co-depended systems and sub-systems, there is a chain reaction of possible failures that engineers can trace back and find the cause of failure. For example, a failure in the Cooling Water System could affect critical components associated with the Main Engine, which would have a knock-on effect on other interconnected components. A high-temperature reading of the main engine could indicate a Cooling Water System failure but could also be a result of a failure in the Lubricating Oil System.

The system administrator sets up varying scenarios, in which a combination of appropriate data values such as temperature and pressure readings, along with audio/vibration signals are used across several interconnected systems to represent a particular mechanical failure. A list of possible scenarios and how a hypothetical failure could be developed was put together by engineers working in this project. It was vital during the usability test where VR user could explore and identify virtual failures using familiar techniques they usually do in-situ.

Handheld controllers allow the user to navigate around the engine room, through a transport feature, and the ability to select components for examination. When selecting a component, a virtual handheld display attached to one of the controllers shows the available data from the conventional sensors mainly pressure and temperature. Also allows the user to isolate the sound of the selected machinery by muting background sounds for a more focused listening experience.

One of the key features of providing and controlling the data through the server was the ability to include 10-hour data history snapshots. User can go back in time and revisit the state of the machinery and performance along with audio and vibration. Via the remote server, the administrator can control the data of each component in these three categories:

- $\quad$ Current state is the current real-time data when entering the engine room

- Baseline is what is considered to be normal values when in working condition

- History is the state of the component up to ten hours before. It enables the user to have a good overview when signs of failure might occur or how they developed within in the past ten-hour window and how might be associated with other changes of other machinery within the engine room.

Audio features and thus, vibrations are implemented similarly. The list below shows the level of audio control and volume for each machinery. The different audio types are represented as numbers in the graph with increments of (0.5) in a range between (0) and (3). See Fig. 4.

- $\quad$ Volume (ranging from 0.0 to 2.0 )

- Audio Type

$\circ \quad$ None $(0)$

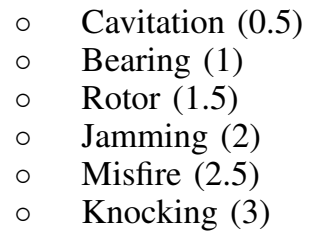

- $\quad$ Fault-tolerant Control-fidelity

$\begin{array}{ll}\circ & \text { Very Low (0) } \\ \circ & \text { Low (1) } \\ \circ & \text { Medium (2) } \\ \circ & \text { High (3) } \\ \circ & \text { Severe (4) }\end{array}$

The type of audio and the degree of fidelity can change accordingly to provide a realistic representation of the failure. The fault fidelity parameter is the amount of blending between the clean (healthy) and the faulty audio signal. For example, depending on the failure scenario pushed forward during the user testing, the knocking audio type failure began with a low fault fidelity six hours earlier. Every hour there is an increase of the fault fidelity level from low to severe, see Fig. 5.

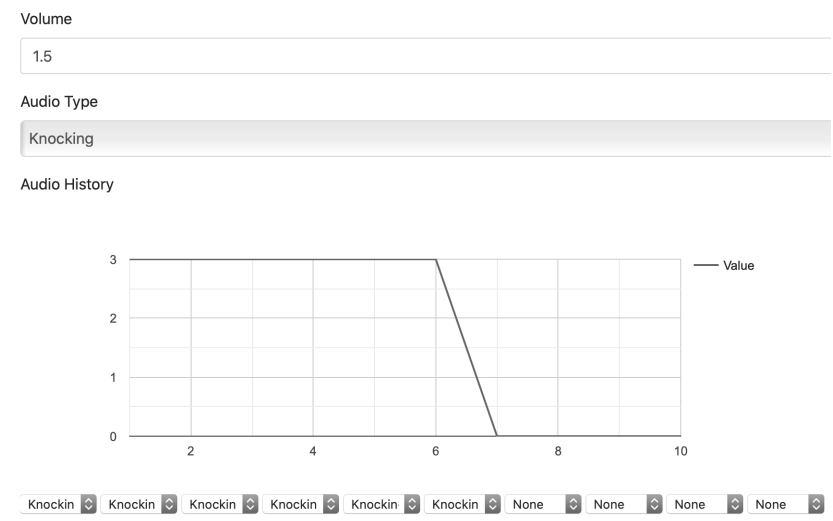

Fig. 4. Volume and Audio Type shows the current state of machinery. Audio History provides information when the failure has started and the type of audio failure. Y-axis represents the type of audio the $\mathrm{X}$-axis the hours.

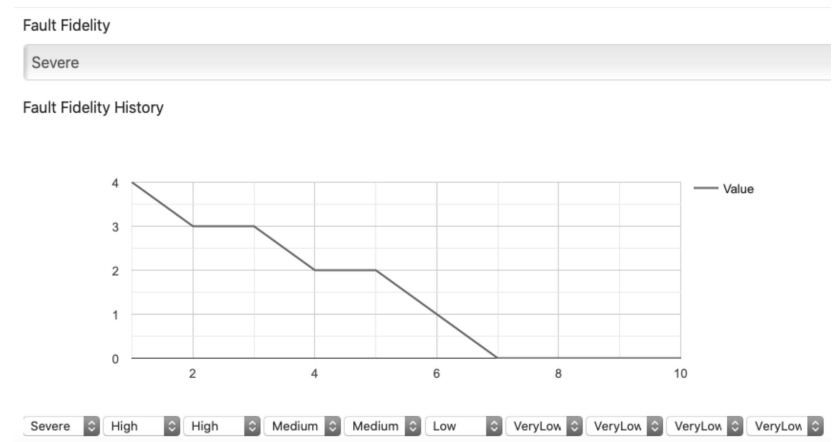

Fig. 5. The graph shows the fault fidelity history as it develops at hourly intervals. The increase of fault fidelity is shown on the Y-axis of the graph in increments of one. Very low is represented with zero and Severe with four.

The user can view current and past data of selected machinery with a handheld display within the simulator. The virtual data display is attached to one of the controllers, and the user can raise the hand towards the headset to view the available data. In addition, users can isolate the sound of 
selected machinery for focused listening as well as exclude the selected machinery from the overall audio to listen to the surrounding environment alone. Fig. 6 shows the handheld virtual display.

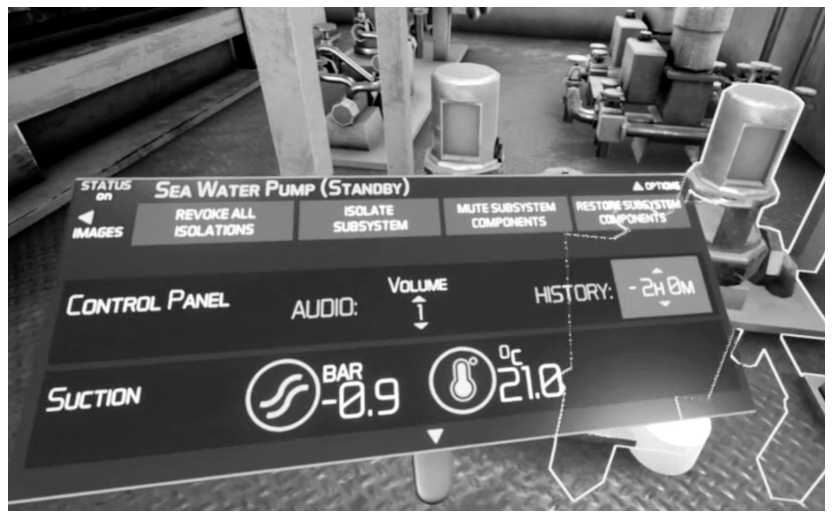

Fig. 6. Handheld virtual displace showing the selected machinery Sea Water Pump(Standby).

\section{USER TESTING}

Seven marine engineers took part in the usability testing with an average of 27 years of experience. Six participants were Chief Engineers, and one was Second Engineer with two years of experience. A set of performing task scenarios aimed to determine the usability of the VR system for engineers with different levels and types of experience. Mainly to access how marine engineers were able to diagnose system failures using the simulation and examine to what extend audio feedback plays a role in that process. The signal was spatialised using a binaural encoder for built-in HTC VIVE Pro HMD headphones and played simultaneously on a four-channel (4.0) arrangement. The approach enabled users to choose to listen to either the loudspeaker based audio, or the headphone-based audio, and get spatial cues from each. Engineers were first familiarised with the system, without any simulated faults, in order to ensure that they were able to navigate around the virtual space and access the data through the controllers. After a period of familiarisation, each participant went through the four simulated scenarios. Two scenarios involve simulated faults, and two had no fault. The four scenarios were presented in a randomised order. Participants were not told whether or not there would be faults present but were asked to undertake a system health check of the simulated engine room as if they were undertaking a routine check. They were asked to assess whether or not they felt there was a potential system failure, what might have caused the failure and possible further actions or if the system was operating normally. Participants were encouraged to 'think aloud' during the familiarisation and while taking the test.

\section{RESUlTs AND Discussion}

The focus of the usability test was to identify how engineers felt regarding the system and if they believe from their experience that such a system could be applied in a MASS scenario. This includes the use as diagnostic and teaching tool from a remote location, in what ways the virtual environment and user interface were useful, their perception of the level of immersion, and what improvements or alternative uses they felt the system could have.

Four out of the seven engineers were able to identify if something was wrong and what might have caused failure but stated that they would need more time to investigate further in order to identify the exact type. The other three participants diagnosed the correct failure type. All interviews were audio-recorded and transcribed and analysed using a Qualitative Data Analysis (QDA) process. Transcriptions were checked for technical terms in case of transcription errors, and interviewer's comments were removed from the analysis to focus on respondent comments. Responses from the interviews were coded into three key categories:

- Features (discussion of specific features in the system)

- System Potential (what the system could be used for, and how it could be used)

- User Experience (usability of the system)

The overall response firmly focused around the immersive features. 'Sound' was discussed in $53.9 \%$ of coded phrases, 'vibration' in $21.7 \%$ and 'immersion' in $14.8 \%$ and 'data' in $10.2 \%$. While we acknowledge that the focus of this project was around the implementation of audio, and is interesting to see such results, we assume that the high percentage of 'sound' was due to the alternative ways of how sound is presented to them otherwise impossible to do so in the engine room. For example, the ability to isolate the sound of machinery from the background noise.

The following are comments for the users during the interview stage after caring out the usability tests.

I found myself doing what I do in the engine room when I was listening to the pumps [...] I was moving my head to look up so I wasn't concentrating on the machinery. $(\mathrm{P} 3)$

Even though you see the data [...] you are more convinced that something has gone wrong through sound rather than the data.(P1)

You can actually listen to the machine and it's sounding good cos if there's a problem they sound a hundred percent different.(P6)

I'm not saying you can use the sound for everything but sound is one of the key components. (P7)

The time history was listed as a particularly positive feature, as it allows engineers to very quickly identify and compare any changes in both audio and the data. Engineers mentioned that changes in audio from upcoming failures would occur before a significant change in the system's temperature or pressure is registered or trigger the alarm's threshold levels. As a result, the ability to check the history of machinery provides a broader picture and able to form the appropriate action.

You know because if you can identify a future failure you're saving a lot of money because if you don't you could write off an engine.(P5) 
The sound is quite important. It leads you to the fact-if you're looking at pressures and the pressures are fine and the temperatures are fine, but the noise has changed, you know that something is up, and that is the trigger to start finding down the right route. $(\mathrm{P} 3)$

There were also some negative statements regarding the fidelity of the system data and immersion. One user points out that even though both compressors seemed to be switched off, with room temperature reading, at least one should have retained some heat as it had been working earlier.

In terms of user experience, the overall focus was on positive aspects. Users found the system to be generally easy to navigate and stated that they very quickly adapted to the simulation. Users identified the system as ideal for problem-solving and particularly liked the integration of the sound and vibration with data. With a controlled test environment, we were able to identify how a hypothetical system might be presented and how users may anticipate the interaction. Overall the result showed a positive outcome in terms of the efficiency, controllability and understanding of the possibilities. Even though audio for the simulated faults gone through a processing stage, none of the users mentions any noticeable difference.

Concerns were raised regarding the use of the system. Mainly the lack of familiarity of interface and controls. As expected, some users were more competent in using the controllers. Even though competition time was not considered as part of this research, there was a noticeable difference between the young engineer, with only two-year experience, and Chief Engineers. Data sample is small and cannot provide any concrete claims regarding why the younger inexperienced engineer was able to identify the failure faster than engineers with more experience. However, we suspect that the new generation of engineers have been exposed to a broader gaming diet and able to grasp the concept of navigating and immersion faster. Besides, the young engineer was the only user with previous experience with VR systems which can potentially factor into the learning curve of the simulation.

When analysing statements which expressed opinion, $74.9 \%$ of coded statements were 'positive' in focus. When questioned about the system potential for remote diagnosis through audio, all respondents gave overall positive feedback regarding the potential use in MASS industry. Also, some participants saw a wider potential as a training tool, where data from a real ship can be used to familiarise new employers and provide regular training for less common failures.

A new employee could come in and they would know what they are supposed to be looking for. If I was to join a new ship, it would come in handy to me. You could have a simulated breakdown. You could have someone in VR in the machinery spaces. $(\mathrm{P} 1)$

All training works best with a blended solution. In my head..the best training is with physical equipment - but then I'm old school. If you can supplement that or bring stuff along- side it that is good.(P4)

The ability to identify failures and the possible cause of the problem under time-constrained circumstances on an unfamiliar vessel, suggests that the virtual environment and the integration of real-time immersive features offer significant potential for enhancing remote diagnostics. A key statement from several participants was that they did not listen for specific characteristics, but the change of audio. It allowed engineers to quickly identify changes in both audio/vibration and the data.

The User Experience Questionnaire (UEQ) conducted after the test aims towards understanding an overall impression of user experience. The questionnaire consists of 26 pairs of different attributes that may apply to the system. Participants express their agreement with the attributes by selecting the appropriate box that most closely reflects their impression about their experience with the system. Both classical usability aspects (efficiency, perspicuity, dependability) and user experience aspects (originality, stimulation) are measured. Fig. 7 shows users responses on a seven-point Likert scale between -3 and +3 . While this is not a unified representation of the system due to the limited users available, it is, however, an early indication where those user experienced attributes might need improvement and consideration.

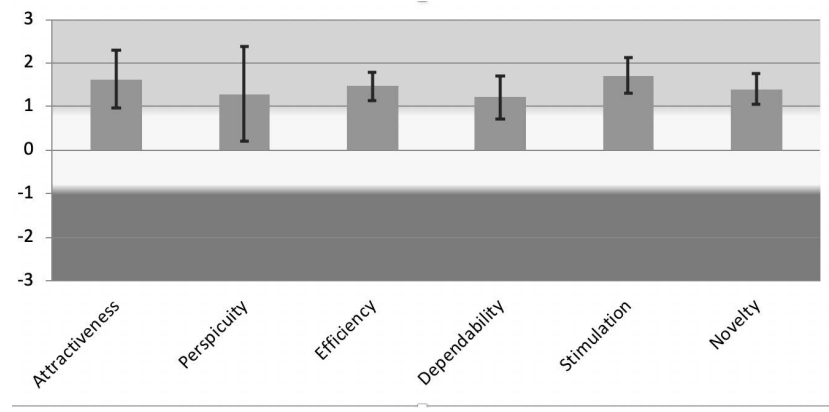

Fig. 7. Data from users completing the UEQ on a seven-point Likert scale between -3 and +3 .

\section{CONCLUSION}

The engine room is a complex and active system which is hard to replicate. Information like smell, heat and whole-body vibration proved to be impossible to reproduce precisely within the scope of this project. Interviews with experienced engineers identify ways and scenarios in which they identify current and future failures in a vessel's engine room. The project developed a VR simulator on exploring how sound qualities can be enhanced and even exceed the missing sensory information. In a remote location, the sound can be analysed and provide focused information to the participant. Engineers often rely on their senses to validate the sensor data that is presented to them in a real engine room. As a result, it is essential to make available vital audiovisual and haptic information for users to enable a realistic and immersive environment.

We recognise that due to excessive sounds in the engine room, vital information is often missed. Even though data from machinery can provide a good overview of the system, they are not always reliable. The knowledge and experience of an engineer play a key role in identifying failures, including anticipating upcoming failures. The perception of sound experience in a virtual environment can be enhanced and refined to provide 
the best possible experience and information to the user. Our research showed that the use of a remote server was vital to emulated data feed as if it was a real vessel. This includes being able to control the volume, the ability to filter out any unwanted surrounding noise, focus on particular machinery, and to be able to scroll back in time and look at the data, including audio, from ten hours before. User perceives the simulation as immersive with audio and vibration playing a key role. With the advances of sound techniques, a live audio feed can be analysed and transmitted to a remote location providing a holistic view of the situation. For MASS, the industry needs to invest on how to distribute the cognition overload and new human-computer and human-human challenges of engineers and the possibly multiple engineers working remotely on the same vessel from different remote control centres [18], [19]. We need to develop new and innovate ways in which audio and data can be transmitted from the vessel to the control centre. The presented project is a practical case study on how future real-time audio feeds can be applied to enhance remote operation and engine room diagnostics in the MASS industry.

\section{REFERENCES}

[1] J. Louro, P. Vazquez and R. M. de la Campa, "Accident Risk Factors in Conventional and High Speed Ferry Ships in Spain". in Journal of Maritime Research. Vol.3-8, Dec. 2012.

[2] A. Dahlgren, w. Van Leeuwen, A. Kircher, M. Lutzhoft, M. Barnett, G. Kecklund, and T. Akerstedt, "Sleep and fatigue in bridge officers working $6 \mathrm{~h}$ on and $6 \mathrm{~h}$ off - a simulator study", Journal of Sleep Research, (21) 331, 2012.

[3] L. Turchet, G. Fazekas, M. Lagrange, H. S. Ghadikolaei and C. Fischione, "The Internet of Audio Things: state-of-the-art, vision, and challenges", IEEE Internet of Things Journal, Web: https://doi.org/10.1109/JIOT.2020.2997047.

[4] J. Kuschel and F. Ljungberg, "Decentralized Remote Diagnostics: A Study of Diagnostics in the Marine Industry", in S. Fincher, P. Markopoulos, D. Moore, R. Ruddle (eds). People and Computers XVIII - Design for Life. 2005.

[5] G. Meadow, C. Barlow, T. Michalidis, S. Bracagna, L. Arrigoni, E. Broadhurst and E. Rajabally, "IMAGINE Work Package 1". Tech. Report. Rolls- Royce/Solent University, 2017, Unpublished.

[6] M. Fachot, "Virtual reality is central to aviation and maritime training", IEC e- tech, Issue 4, 2016, Web: https://iecetech.org/issue/2016-04.

[7] T. Hogg and S. Ghosh, "Autonomous merchant vessels: examination of factors that impact the effective implementation of unmanned ships", Australian Journal of Maritime Ocean Affairs, Vol. 8:3, 2016, pp. 206222. Web: https://doi.org/10.1080/18366503.2016.1229244.

[8] B. Resch, A. Wichmann and N. Gll, "Usability in 4D AR: Visualising Multi- temporal Real-time Geo-data in Augmented Reality Environments", International Journal of Interactive Mobile Technologies (iJIM) 9(4), Jan. 2015, pp. 23-33.

[9] K. Kilteni, R. Groten and M. Slater, "The sense of embodiment in virtual reality", Presence: Teleoperators and Virtual Environments, Vol. 21(4), 2012, pp. 373-387.

[10] C. Barlow, T. Michailidis, G. Meadow, J. Gouch and E. Rajabally, "Using Immersive Audio and Vibration to Enhance Remote Diagnosis of Mechanical Failure in Uncrewed Vessels", in AES International Conference on Immersive and Interactive Audio, Mar. 2019. Web: http://www.aes.org/e-lib/browse.cfm?elib=20428.

[11] M. Schrepp, A. Hinderks and J. Thomaschewski, "Construction of a benchmark for the User Experience Questionnaire (UEQ)", International Journal of Interactive Multimedia and Artificial Intelligence, Vol. 4, No. 4, 2017, pp. 40-44.

[12] Y. Man, M. Lundh and S. N. MacKinnon, "Managing unruly technologies in the engine control room: from problem patching to an architectural thinking and standardization". WMU Journal of Maritime Affairs Vol. 17, 2018, pp. 497-519. Web: https://doi.org/10.1007/s13437-0180159 -y.
[13] W. J. Lee, H. Wu, H. Yun, H. Kim, M. B.G. Jun and J. W Sutherland, "Predictive Maintenance of Machine Tool Systems Using Artificial Intelligence Techniques Applied to Machine Condition Data", Procedia CIRP, Vol. 80, 2019, pp. 506 - 511. Web: https://doi.org/10.1016/j.procir.2018.12.019.

[14] M. Iber, P. Lechner, C. Jandl, M. Mader and M. Reichmann, "Auditory augmented process monitoring for cyber physical production systems", Personal and Ubiquitous Computing, 2020. Web: https://doi.org/10.1007/s00779-020-01394-3

[15] J. Lee, H. Choi, D. Park, Y. Chung, H. Y. Kim and S. Yoon, "Fault Detection and Diagnosis of Railway Point Machines by Sound Analysis", Sensors, Vol. 16, No. 4: 549, 2016. Web: https://doi.org/10.3390/s16040549.

[16] D. Goyal and B. S. Pabla, "The Vibration Monitoring Methods and Signal Processing Techniques for Structural Health Monitoring: A Review", Archives of Computational Methods in Engineering. Vol. 23, 2016, pp. 585-594. Web: https://doi.org/10.1007/s11831-015-9145-0.

[17] M. Schrepp, A. Hinderks, and J. Thomaschewski, "Applying the User Experience Questionnaire (UEQ) in Different Evaluation Scenarios" in A. Marcus (Ed.) Design, User Experience, and Usability. Theories, Methods, and Tools for Designing the User Experience. Lecture Notes in Computer Science, Vol. 8517, 2014, pp. 383-392.

[18] E. Hutchins and T. Klausen, "Distributed cognition in an airline cockpit", Cognition and communication at work. 1996. pp, 15-34

[19] Y. Rogers, "New Theoretical Approaches for Human-Computer Interaction", Annual Review of Information Science and Technology". Vol. 38, 2004. 